\title{
SOME IMPLICATIONS OF THE EULER-POINCARÉ CHARACTERISTIC FOR COMPLETE INTERSECTION MANIFOLDS
}

\author{
BANG-YEN CHEN $^{1}$ AND KOICHI OGIUE ${ }^{2}$
}

\begin{abstract}
Studies on relations between Euler-Poincaré characteristic and codimension of a complete intersection manifold in a complex projective space.
\end{abstract}

1. Introduction. Let $P_{n+p}(C)$ be an $(n+p)$-dimensional complex projective space. An $n$-dimensional algebraic manifold $M$ imbedded in $P_{n+p}(C)$ is called a complete intersection manifold if $M$ is given as an intersection of $p$ nonsingular hypersurfaces $M_{1}, \cdots, M_{p}$ in general position in

$$
P_{n+p}(C): M=M_{1} \cap \cdots \cap M_{p} .
$$

It is known that the Chern classes of a complete intersection manifold $M$ are completely determined by the degrees of $M_{1}, \cdots, M_{p}$. In particular, the Euler-Poincare characteristic of a complete intersection manifold is completely determined by the degrees of $M_{1}, \cdots, M_{p}$.

In $\$ 2$ we prove a formula for the Euler-Poincaré characteristic of a complete intersection manifold in terms of the degrees.

It is sometimes very important to know the smallest codimension for a complete intersection manifold: the smallest $p$ for which $M$ can be imbedded as a complete intersection manifold in $P_{n+p}(C)$. In $\S 3$ we prove several results in this direction.

2. The Euler-Poincaré characteristic of complete intersection manifolds. Let $P_{n+p}(C)$ be an $(n+p)$-dimensional complex projective space and let $M$ be an $n$-dimensional complete intersection manifold imbedded in $P_{n+p}(C): M=M_{1} \cap \cdots \cap M_{p}$, where the $M_{\alpha}$ 's are nonsingular hypersurfaces in $P_{n+p}(C)$. The following theorem gives a concrete formula for the Euler-Poincaré characteristic of a complete intersection manifold.

Received by the editors April 17, 1973 and, in revised form, August 31, 1973.

AMS (MOS) subject classifications (1970). Primary 14M10, 57D20.

Key words and phrases. Euler-Poincaré characteristic, complete intersection manifold, complex projective space, codimension.

1 Work done under partial support by NSF Grant 36684.

${ }^{2}$ Work done under partial support by the Matsunaga Science Foundation. 
THEOREM 2.1. ${ }^{3}$ Let $M=M_{1} \cap \cdots \cap M_{p}$ be an $n$-dimensional complete intersection manifold imbedded in $P_{n+p}(C)$. If $\operatorname{deg} M_{\alpha}=a_{\alpha}$, then the EulerPoincaré characteristic $\chi(M)$ of $M$ is given by

$$
\chi(M)=\left[\sum_{k=0}^{n}(-1)^{k}\left(\begin{array}{c}
n+p+1 \\
n-k
\end{array}\right) \sigma_{k}\right]\left(\prod_{\alpha=1}^{p} a_{\alpha}\right),
$$

where $\sigma_{k}=\sum_{\alpha_{1} \leqq \ldots \leqq \alpha_{k}} a_{\alpha_{1}} a_{\alpha_{2}} \cdots a_{\alpha_{k}}$ (the sum of all homogeneous monomials of degree $k$ in $\left.a_{1}, a_{2}, \cdots, a_{p}\right)$ and $\left(\begin{array}{c}n+p+1 \\ n-k\end{array}\right)$ is the binomial coefficient.

Proof. Let $\tilde{h}$ be the generator of $H^{2}\left(P_{n+p}(C), Z\right)$ corresponding to the divisor class of a hyperplane $P_{n+p-1}(C)$. Then the total Chern class $c\left(P_{n+p}(C)\right)$ of $P_{n+p}(C)$ is given by

$$
c\left(P_{n+p}(C)\right)=(1+\tilde{h})^{n+p+1}
$$

Let $j: M \rightarrow P_{n+p}(C)$ be the imbedding and $v$ be the normal bundle of $j(M)$ in $P_{n+p}(C)$. Then the total Chern class $c(v)$ of $v$ is given by

$$
c(v)=\left(1+a_{1} h\right) \cdots\left(1+a_{p} h\right),
$$

where $h$ is the image of $\tilde{h}$ under the homomorphism $j^{*}: H^{2}\left(P_{n+p}(C), Z\right) \rightarrow$ $H^{2}(M, Z)$. Since $j^{*} T\left(P_{n+p}(C)\right)=T(M) \oplus v$ (Whitney sum), we have

$$
j^{*} c\left(P_{n+p}(C)\right)=c(M) \cdot c(v),
$$

where $c(M)$ is the total Chern class of $M$. Let $c_{i}(M)$ be the $i$ th Chern class of $M$. Then we have

$$
(1+h)^{n+p+1}=\left[1+c_{1}(M)+\cdots+c_{n}(M)\right] \cdot\left(1+a_{1} h\right) \cdots\left(1+a_{p} h\right)
$$

which implies that

$$
c_{n}(M)=\left[\sum_{k=0}^{n}(-1)^{k}\left(\begin{array}{c}
n+p+1 \\
n-k
\end{array}\right) \sigma_{k}\right] h^{n} .
$$

Taking the values of both sides on the fundamental cycle of $M$, we have

$$
\chi(M)=\left[\sum_{k=0}^{n}(-1)^{k}\left(\begin{array}{c}
n+p+1 \\
n-k
\end{array}\right) \sigma_{k}\right]\left(\prod_{\alpha=1}^{p} a_{\alpha}\right) .
$$

Remark. Let $b_{i}(M)$ be the $i$ th Betti number of $M$. It is known that if $M$ is an $n$-dimensional complete intersection manifold, then

$$
\begin{aligned}
b_{2 k}(M) & =1 & & (2 k \neq n), \\
b_{2 k+1}(M) & =0 & & (2 k+1 \neq n) .
\end{aligned}
$$

${ }^{3}$ Although Theorem 2.1 can be obtained from Theorem 22.1.1 of [1], we give here a direct proof for the sake of completeness. 
Therefore $\chi(M)=\sum_{i=0}^{2 n}(-1)^{i} b_{i}(M) \geqq n+1 \quad$ (resp. $\left.\leqq n+1\right)$ provided that $n$ is even (resp. odd).

3. Some implications of the Euler-Poincaré characteristic. First we prove the following:

THEOREM 3.1. Let $M$ be an $n$-dimensional complete intersection manifold. If $\chi(M)=v_{1} \cdots v_{p}$ for some prime numbers $v_{1}, \cdots, v_{p}(\neq \pm 1)$, then $M$ can be imbedded as a complete intersection manifold in $P_{n+p}(C)$.

Proof. We can assume without loss of generality that $M$ is a complete intersection manifold imbedded in $P_{n+q}(C)$ for some $q \geqq p$. In fact, if $M$ is a complete intersection manifold imbedded in $P_{n+r}(C)$ for some $r<p$, then, by imbedding $P_{n+r}(C)$ into $P_{n+q}(C)$ as a linear subspace for some $q \geqq p, M$ can be considered as a complete intersection manifold imbedded in $P_{n+q}(C)$.

Theorem 2.1 implies that $\chi(M)$ is given as a product of $q+1$ integers: $\chi(M)=[\cdots] a_{1} \cdots a_{q}$. On the other hand, since $\chi(M)=v_{1} \cdots v_{p}$, at least $q-p a_{\alpha}$ 's must be equal to 1 . This implies that $M$ can be imbedded as a complete intersection manifold in some $(n+p)$-dimensional linear subspace $P_{n+p}(C)$ in $P_{n+q}(C)$. Q.E.D.

It is sometimes very important to know the smallest codimension for which $M$ can be imbedded as a complete intersection manifold. From this point of view, Theorem 3.1 may not be the best possible in general. We shall prove several partial improvements of Theorem 3.1 in the following:

First we prove the following:

Lemma 3.2. Let $M$ be an n-dimensional complete intersection manifold imbedded in $P_{n+p}(C)$. Assume that $\chi(M)=v_{1} \cdots v_{p}$ for some prime numbers $v_{1}, \cdots, v_{p}(\neq \pm 1)$. If the Diophantine equation $\sum_{k=0}^{n}(-1)^{k}\left(\begin{array}{c}n+p+1 \\ n-k\end{array}\right) \sigma_{k}=$ \pm 1 has no solution satisfying $a_{1} \geqq 2, \cdots, a_{p} \geqq 2$, then $M$ can be imbedded as a complete intersection manifold in $P_{n+p-1}(C)$.

Proof. Theorem 2.1 implies that $\chi(M)$ is given as a product of $p+1$ integers: $\chi(M)=[\cdots] a_{1} \cdots a_{p}$. On the other hand, since $\chi(M)=$ $v_{1} \cdots v_{p}$, at least one among [ $\left.\cdots\right], a_{1}, \cdots, a_{p}$ must be equal to \pm 1 . If the Diophantine equation $[\cdots]= \pm 1$ has no solution satisfying $a_{1}>1, \cdots, a_{p}>1$, then at least one $a_{\alpha}$ must be equal to 1. Q.E.D.

THEOREM 3.3. Let $M$ be a complete intersection manifold. If $\chi(M)$ is a prime number, then $M$ is one of the following:

(1) a linear subspace

(2) a quadric in $P_{2}(C)$,

(3) a curve of degree 4 in $P_{2}(C)$. 
Proof. In consideration of Theorem 3.1, we may assume that $M$ is a nonsingular hypersurface of $P_{n+1}(C)$. Putting $a=a_{1}$, from Theorem 2.1 we have

$$
\chi(M)=\left[\sum_{k=0}^{n}(-1)^{k}\left(\begin{array}{l}
n+2 \\
n-k
\end{array}\right) a^{k}\right] a=\frac{(1-a)^{n+2}-1+(n+2) a}{a^{2}} \cdot a .
$$

Since $\chi(M)$ is a prime number, either $a=1$ or

$$
\frac{(1-a)^{n+2}-1+(n+2) a}{a^{2}}= \pm 1 .
$$

If $a=1$, then $M$ is a linear subspace. Therefore we consider the latter case. It suffices to prove the following:

LeMma. The only positive integral solutions for the Diophantine equation $(1-a)^{n+2}-1+(n+2) a= \pm a^{2}$ are $(n, a)=(1,2)$ and $(n, a)=(1,4)$.

Proof of lemma. Since $a=1$ cannot be a solution for the equation $(1-a)^{n+2}-1+(n+2) a= \pm a^{2}$, we consider this equation for $a \geqq 2$. Let

$$
f_{n}(a)=\frac{(1-a)^{n+2}-1+(n+2) a}{a^{2}} \text { for } a \geqq 2 .
$$

(i) If $n$ is even: It is easy to show that $f_{n}(a)$ is monotonically increasing and $f_{n}(2)=(n+2) / 2>1$. Therefore the equation $(1-a)^{n+2}-1+(n+2) a=$ $\pm a^{2}$ has no solution of the form $(n, a)=($ even, $*)$.

(ii) If $n$ is odd: $f_{n}(a)$ is monotonically decreasing and $f_{n}(2)=(n+1) / 2$. It is also easy to show that $f_{n}(5) \leqq-2$. Therefore the only candidates for the solutions for the equation $(1-a)^{n+2}-1+(n+2) a= \pm a^{2}$ are of the form $(n, a)=(*, 2),(n, a)=(*, 3)$ or $(n, a)=(*, 4)$. We can easily prove that the only solutions are $(n, a)=(1,2)$ and $(n, a)=(1,4)$. Q.E.D.

COROLlary 3.4. Let $M$ be a complete intersection manifold. If $\operatorname{dim} M>1$ and if $\chi(M)$ is a prime number, then $M$ is a linear subspace.

THEOREM 3.5. Let $M$ be a complete intersection manifold. If $\operatorname{dim} M>1$ and if $\chi(M)=v_{1} \nu_{2}$ for some prime numbers $v_{1}, v_{2}(\neq \pm 1)$, then $M$ can be imbedded as a hypersurface.

Proof. In consideration of Theorem 3.1, we may assume that $M$ is imbedded as a complete intersection in $P_{n+2}(C)$, where $n=\operatorname{dim} M$. From Theorem 2.1, we have

$$
\chi(M)=\left[\sum_{k=0}^{n}(-1)^{k}\left(\begin{array}{l}
n+3 \\
n-k
\end{array}\right) \sigma_{k}\right] a_{1} a_{2} .
$$


We consider the Diophantine equation

$$
\sum_{k=0}^{n}(-1)^{k}\left(\begin{array}{l}
n+3 \\
n-k
\end{array}\right) \sigma_{k}= \pm 1
$$

Multiplying both sides of (3.1) by $a_{1}-a_{2}$, we obtain

$$
\sum_{k=0}^{n}(-1)^{k}\left(\begin{array}{l}
n+3 \\
n-k
\end{array}\right)\left(a_{1}^{k+1}-a_{2}^{k+1}\right)= \pm\left(a_{1}-a_{2}\right),
$$

which can be written as

$$
\begin{aligned}
-\left[\sum_{k=0}^{n+1}(-1)^{k}\left(\begin{array}{c}
n+3 \\
n+1-k
\end{array}\right) a_{1}^{k}\right] & \\
& +\left[\sum_{k=0}^{n+1}(-1)^{k}\left(\begin{array}{c}
n+3 \\
n+1-k
\end{array}\right) a_{2}^{k}\right]= \pm\left(a_{1}-a_{2}\right) .
\end{aligned}
$$

Hence we have

$$
\begin{aligned}
-\frac{\left(1-a_{1}\right)^{n+3}-1+(n+3) a_{1}}{a_{1}^{2}} & \\
+\frac{\left(1-a_{2}\right)^{n+3}-1+(n+3) a_{2}}{a_{2}^{2}} & = \pm\left(a_{1}-a_{2}\right)
\end{aligned}
$$

or

$$
\begin{aligned}
\frac{\left(1-a_{1}\right)^{n+3}-1+(n+3) a_{1}}{a_{1}^{2}} & \pm a_{1} \\
& =\frac{\left(1-a_{2}\right)^{n+3}-1+(n+3) a_{2}}{a_{2}^{2}} \pm a_{2}
\end{aligned}
$$

Let

$$
f_{n}(a)=\frac{(1-a)^{n+3}-1+(n+3) a}{a^{2}} \pm a .
$$

Then it is not difficult to prove that

$$
\begin{array}{ll}
f_{n}^{\prime}(a)>0 & \text { for } a \geqq 3 \\
f_{n}^{\prime}(a)<0 & \text { for } a \geqq 2 \text { if } n \text { is odd, }
\end{array}
$$

This implies that $f_{n}(a)$ is monotonically increasing (resp. decreasing) for $a \geqq 3$ (resp. $a \geqq 2$ ) if $n$ is odd (resp. even). Therefore from (3.2) we deduce that either $a_{1}=a_{2}$ or $f_{n}(2)=f_{n}(*)$, the latter case arising only when $n$ is odd. It is easy to show that the only solution for the latter case is $f_{1}(2)=f_{1}(3)$. But this is excluded by the assumption that $n>1$. Hence we have $a_{1}=a_{2}$. 
Putting $a=a_{1}=a_{2}$, from (3.1) we have

Let

$$
\sum_{k=0}^{n}(-1)^{k}(k+1)\left(\begin{array}{l}
n+3 \\
n-k
\end{array}\right) a^{k}= \pm 1
$$

$$
g_{n}(a)=\sum_{k=0}^{n}(-1)^{k}(k+1)\left(\begin{array}{l}
n+3 \\
n-k
\end{array}\right) a^{k} \mp 1 .
$$

Then we have $f_{n}^{\prime}(a)=-g_{n}(a)$, which, together with (3.3), implies that

$$
\begin{array}{lll}
g_{n}(a)<0 & \text { for } a \geqq 3 & \text { if } n \text { is odd, } \\
g_{n}(a)>0 & \text { for } a \geqq 2 & \text { if } n \text { is even. }
\end{array}
$$

Therefore the only candidate for the solution for (3.4) is $(n, a)=($ odd, 2$)$. But, since $g_{n}(2)=-f_{n}^{\prime}(2)=\mp 1$ if $n$ is odd, (3.4) has no solution of this form.

Thus we have proved that the Diophantine equation (3.1) has no solution satisfying $n>1, a_{1} \geqq 2$ and $a_{2} \geqq 2$. This, combined with Lemma 3.2, implies that $M$ can be imbedded as a hypersurface. Q.E.D.

We have excluded the 1-dimensional case in Theorem 3.4. The following result gives a solution for this case.

THEOREM 3.6. Let $M$ be a complex curve which is a complete intersection manifold. If $\chi(M)=v_{1} \cdots v_{p}$ for some prime numbers $v_{1}, \cdots, v_{p}$ $(\neq \pm 1)$, then $M$ can be imbedded as a complete intersection manifold in $P_{p}(C)$ except when

$$
\begin{aligned}
& P_{3}(C) \supset M=M_{1} \cap M_{2} \quad\left(\operatorname{deg} M_{1}=2, \operatorname{deg} M_{2}=3\right), \\
& P_{4}(C) \supset M=M_{1} \cap M_{2} \cap M_{3} \quad\left(\operatorname{deg} M_{\alpha}=2\right) .
\end{aligned}
$$

Proof. In consideration of Theorem 3.1, we may assume that $M$ is imbedded as a complete intersection manifold in $P_{1+p}(C)$. From Theorem 2.1 we have

$$
\chi(M)=\left[p+2-\sum a_{\alpha}\right] a_{1} \cdots a_{p}
$$

so that $\chi(M)$ is a product of $p+1$ integers. Therefore at least one of them must be equal to \pm 1 . It is easy to prove that the equation $p+2-\sum a_{\alpha}=$ \pm 1 has solutions satisfying $a_{1} \geqq 2, \cdots, a_{p} \geqq 2$ only when $p=2$ or $p=3$, and the solutions are, respectively, $\left\{a_{1}, a_{2}\right\}=\{2,3\}$ or $\left\{a_{1}, a_{2}, a_{3}\right\}=$ $\{2,2,2\}$. Q.E.D.

For complex surfaces we have the following:

THEOREM 3.7. Let $M$ be a complex surface which is a complete intersection manifold. If $\chi(M)=v_{1} \cdots v_{p}$ for some prime numbers $v_{1}, \cdots, v_{p}$ 
$(\neq 1)$, then $M$ can be imbedded as a complete intersection manifold in $P_{1+p}(C)$.

Proof. In consideration of Theorem 3.1, we may assume that $M$ is imbedded as a complete intersection manifold in $P_{2+p}(C)$. From Theorem 2.1 we have

Let

$$
\chi(M)=\left[\left(\begin{array}{c}
p+3 \\
2
\end{array}\right)-\left(\begin{array}{c}
p+3 \\
1
\end{array}\right) \sum a_{\alpha}+\sum_{\alpha_{1} \unlhd_{\alpha_{2}}} a_{\alpha_{1}} a_{\alpha_{2}}\right] a_{1} \cdots a_{p} .
$$

$$
f\left(a_{1}, \cdots, a_{p}\right)=\left(\begin{array}{c}
p+3 \\
2
\end{array}\right)-\left(\begin{array}{c}
p+3 \\
1
\end{array}\right) \sum a_{\alpha}+\sum_{\alpha_{1} \leqq_{\alpha_{2}}} a_{\alpha_{1}} a_{\alpha_{2}} .
$$

Then it is easy to prove that $f\left(a_{1}, \cdots, a_{p}\right) \geqq(p+3) /(p+1)>1$. Therefore at least one of $a_{\alpha}$ must be equal to 1. Q.E.D.

THEOREM 3.8. Let $M$ be a 4-dimensional complete intersection manifold. If $\chi(M)=v_{1} \cdots v_{p}$ for some prime numbers $v_{1}, \cdots, v_{p}(\neq 1)$, then $M$ can be imbedded as a complete intersection manifold in $P_{3+p}(C)$.

PRoof. In consideration of Theorem 3.1, we may assume that $M$ is imbedded as a complete intersection manifold in $P_{4+p}(C)$. From Theorem 2.1, we have

$$
\chi(M)=\left[\sum_{k=0}^{4}(-1)^{k}\left(\begin{array}{l}
p+5 \\
4-k
\end{array}\right) \sigma_{k}\right] a_{1} \cdots a_{p} .
$$

We consider the Diophantine equation

Let

$$
\sum_{k=0}^{4}(-1)^{k}\left(\begin{array}{l}
p+5 \\
4-k
\end{array}\right) \sigma_{k}=1
$$

Then we have

$$
f\left(a_{1}, \cdots, a_{p}\right)=\sum_{k=0}^{4}(-1)^{k}\left(\begin{array}{c}
p+5 \\
4-k
\end{array}\right) \sigma_{k}-1 .
$$

$$
\begin{aligned}
\frac{\partial f}{\partial a_{\alpha}}= & -\left(\begin{array}{c}
p+5 \\
3
\end{array}\right)+\left(\begin{array}{c}
p+5 \\
2
\end{array}\right)\left(a_{\alpha}+\sigma_{1}\right) \\
& -\left(\begin{array}{c}
p+5 \\
1
\end{array}\right)\left(a_{\alpha}^{2}+a_{\alpha} \sigma_{1}+\sigma_{2}\right)+a_{\alpha}^{3}+a_{\alpha}^{2} \sigma_{1}+a_{\alpha} \sigma_{2}+\sigma_{3} .
\end{aligned}
$$

It is easy to show that $f$ has no critical point in $\left\{\left(a_{1}, \cdots, a_{p}\right) \in R^{p} \mid a_{1} \geqq\right.$ $\left.2, \cdots, a_{p} \geqq 2\right\}$. We can also prove, by an induction, that $f>0$ on the boundary of $\left\{\left(a_{1}, \cdots, a_{n}\right) \in R^{p} \mid a_{1} \geqq 2, \cdots, a_{p} \geqq 2\right\}$. These facts imply that $f>0$ on $\left\{\left(a_{1}, \cdots, a_{p}\right) \in R^{p} \mid a_{1} \geqq 2, \cdots, a_{p} \geqq 2\right\}$. Therefore Theorem 3.8 follows from Lemma 3.2. 


\section{BIBLIOGRAPHY}

1. F. Hirzebruch, Neue topologische Methoden in der algebraischen Geometrie, Ergebnisse der Math. und ihrer Grenzgebiete, Heft 9, Springer-Verlag, Berlin, 1956; English transl., Die Grundlehren der math. Wissenschaften, Band 131, SpringerVerlag, New York, 1966. MR 18, 509; 34 \#2573.

Department of Mathematics, Michigan State University, East Lansing, MichIGAN 48823 